\title{
Komplexe Zahnaufbauten als Alternative zur Überkronung
}

\author{
D. Wolff, H. J. Staehle, C. Frese \\ Heidelberg
}

\begin{abstract}
Die Indikationen für direkte Kompositrestaurationen wurden in den letzten Jahren zunehmend erweitert. Die Anwendung von Kompositrestaurationen im Front- und Seitenzahnbereich gehört mittlerweile zur Standardversorgung. Auch ausgedehnte Seitenzahnrestaurationen mit Höckerersatz zeigen sich als erfolgreich im klinischen Einsatz [1]. Im Frontzahnbereich sind ausgedehnte Zahnformkorrekturen und Zahnverbreiterungen aus direkt applizierten Kompositen gute Alternativen zu indirekten Versorgungen, wie zum Beispiel keramischen Veneers oder Kronen [2]. Moderne Kompositmaterialien lassen sich mittels Adhäsivtechnik langzeitstabil an Zahnhartsubstanzen befestigen und weisen dabei hohe mechanische Belastbarkeiten auf. Sie lassen sich zudem ästhetisch anspruchsvoll verarbeiten, da eine breite Palette an Farben und Opazitäten zur Verfügung steht. Im folgenden Fortbildungsartikel werden komplexe Zahnaufbauten aus direkt applizierten Kompositen als Alternative zur Überkronung dargestellt. Es werden anhand von Fallbeispielen technische Vorgehensweisen, Schwierigkeiten bei der Umsetzung, mögliche Komplikationen und Erfolgsraten beschrieben.
\end{abstract}

Das Eingliedern einer hochwertigen zahnärztlichen Restauration, wie zum Beispiel einer Krone oder eines Veneers sollte eine gute parodontale und endodontische Prognose des Zahns voraussetzen. Mancher Zahn weist jedoch eine unsichere Prognose auf, welche das Langzeitüberleben infrage stellt. Oder es findet sich umfangreicher Zahnhartsubstanzverlust wegen kariesoder nicht kariesbedingter Zerstörung, der das Eingliedern einer indirekten Restauration kompromittiert.

Versorgungen werden heute ab einem gewissen Zerstörungsgrad des Zahns in vielen Fällen mit prothetischen Restaurationen durchgeführt. Dies bedeutet Behandlungsaufwand und finanzielle Belastung für den Patienten. Geht der Zahn und somit die Restauration dann doch verloren, so ist mit Unmut über investierte Zeit und gezahltes Geld zu rechnen. In manchen Fällen wären alternative Versorgungen wünschenswert, die gegebenenfalls günstiger und weniger aufwändig sind. Dafür stehen unter anderem langzeitprovisorische Kronen zur Verfügung. Weiterführend ist darüber nachzudenken, ob nicht auch direkte Kompositversorgungen eine alternative Therapie darstellen können.

Ein weiterer wichtiger Aspekt ist die Betrachtung der Erhaltung von Restzahnsubstanz und somit Retentionsflächen für restauratives Material und Restauration. Zähne, die endodontisch behandelt wurden oder die umfangreiche Kariesläsionen bis in den Bereich der Zahnwurzel aufweisen, zeigen nach Behandlung und Exkavation der Karies nicht selten eine starke Aushöhlung der Zahnkrone. Dabei sind orale und vestibuläre Schmelzwände sowie Randleisten eventuell erhalten. Fertigt man an solchen Zähnen eine Präparation für die Aufnahme einer indirekten Krone an, so wird durch den zirkulären Abtrag ein Großteil der noch bestehenden Zahnhartsubstanzen entfernt, sodass am Ende vielleicht nur noch die Wurzel und ein geringer supragingivaler Anteil an Restzahnsubstanz erhalten bleibt. Dies bedeutet eine enorme Schwächung des Zahns. Um der Schwächung des Reststumpfes entgegenzuwirken und eine genügende Retention für die indirekte Krone wiederherzustellen, werden individuelle oder konfektionierte Stifte verwendet und der Zahnstumpf wird mit Aufbaumaterialien rekonstruiert. Anschließend sollte die Präparationsgrenze so platziert werden, dass ein Ferrule-Design gegeben ist. Der Kronenrand sollte den Zahnstumpf $2 \mathrm{~mm}$ im Bereich der gesunden Zahnhartsubstanz überdecken. Durch diesen „Fassreifen“-Effekt erzielt man eine Retention und Stabilität der Krone. Der Ferrule-Effekt ist für die Haltbarkeit der Krone wichtiger, als die Art der Stiftversorgung [3]. Liegt jedoch ein stark ausgehöhlter Zahn, oder ein tief subgingivaler Defekt vor, so wird das Umsetzen dieser Regel schwierig. Es bestehen dann die Möglichkeiten, den Präparationsrand sehr weit nach subgingival zu legen, eine chirurgische Kronenverlängerung oder kieferorthopädische Extrusion durchzuführen, auf das Ferrule-Design (ggf. partiell) zu verzichten, oder die Versorgung 
mit einer Krone zu unterlassen und den Zahn zu extrahieren.

Die klinische Erfahrung bestätigt empirische Daten, wonach unsere Patienten länger ihre Zähne bis ins hohe Alter erhalten und die Prävalenz von unter anderem Wurzelkaries zunimmt [4]. Somit wird der zahnärztliche Behandler zunehmend mit oben beschriebener Fragestellung konfrontiert, wie im Fall tief zerstörter Zähne vorzugehen ist. Natürlich muss für jeden Einzelfall kritisch evaluiert werden, welche der genannten Vorgehensweisen am besten geeignet ist.

Im Folgenden wird die Anfertigung ausgedehnter Kompositrestaurationen zur Versorgung umfangreicher, zum Teil tief subgingivaler Zahnhartsubstanzdefekte beschrieben. Dies erfolgt anhand von 2 Fallbeispielen, jeweils eines im Front- und eines im Seitenzahnbereich.

\section{Fallbeispiel 1}

Interne zweiphasige Restaurationstechnik bei tief subgingivaler Wurzelkaries als Alternative zur Überkronung des Zahns An Zahn 27 mit klinischen Zeichen einer irreversiblen Pulpitis zeigt sich röntgenologisch eine tief subgingivale Wurzelkaries im distalen Bereich ( $\bullet$ Abb. 1). Diese ist bei klinischer Inspektion kaum erkennbar ( $\bullet$ Abb. 2). Nach Applikation von Kofferdam erfolgte die Schaffung einer Zugangskavität von okklusal. Bei der Kariesexkavation wurde zum einen die distale Wand im Sinne einer Tunnelpräparation großflächig penetriert und zum anderen die Pulpa freigelegt. Zunächst wurde nach Einbringen einer Matrize von intern fließähiges Komposit adhäsiv aufgetragen, sodass die distale Wand drucklos geschlossen wurde. Anschließend erfolgte die Vitalexstirpation, Aufbereitung und Obturation der Wurzelkanäle. In einem 3. Schritt wurde die Kavität von okklusal verschlossen.

Die Kariesexkavation erfolgte im Sinne einer modifizierten Tunneltechnik, bei der die distale Randleiste des Zahns erhalten und nur die darunter liegenden und kariös zerstörten Anteile des Zahns entfernt wurden ( $\bullet$ Abb. 3). Diese Technik kann durchgeführt werden, wenn über einen ausreichend einsehbaren Zugang die vollständige Kariesexkavation sichergestellt werden kann. Vorteilhaft ist, dass durch den Erhalt der Randleiste eine größere Stabilität des Zahnes bewahrt wird. Zur Abdichtung des distalen Defekts wurde eine metallische Teilmatrize (Palodent, Dentsply DeTrey, Konstanz) eingeführt, die sich durch die Führung des noch bestehenden Kontaktpunkts und der mit Kofferdam eingeschlagenen Papille gut an den Zahn anlegte ( $\bullet$ Abb. 4). Eine Verkeilung konnte nicht erfolgen, der Keil hätte die Matrize in den Bereich des subgingivalen Zahndefekts gedrückt. Dadurch, dass die Matrize jedoch anatomisch formschlüssig am Zahn anlag,

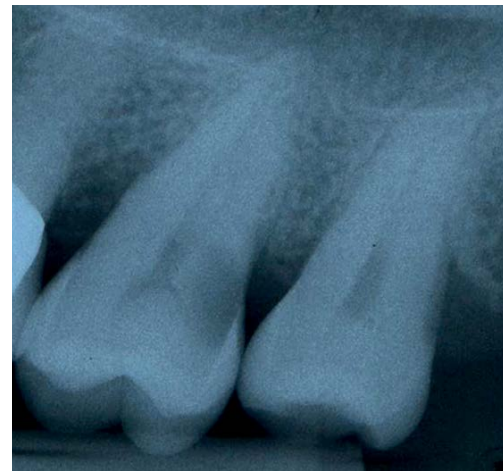

Abb. 1 An Zahn 27 erkennt man eine ausgedehnte distale Wurzelkaries.

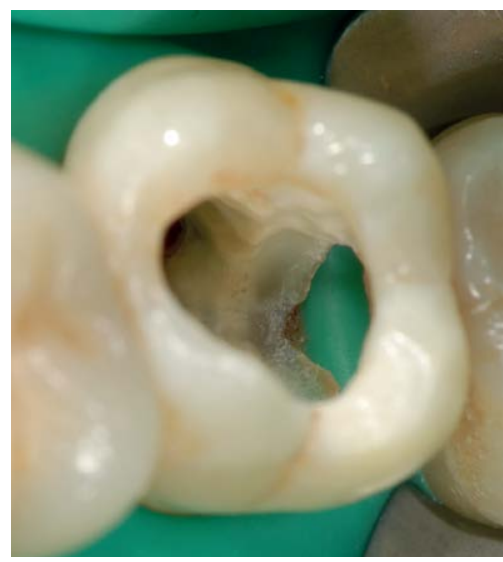

Abb. 3 Zahn 27 nach Anlegen von KofferSinne einer modifizierten Tunneltechnik. dam, Trepanation und Kariesexkavation im

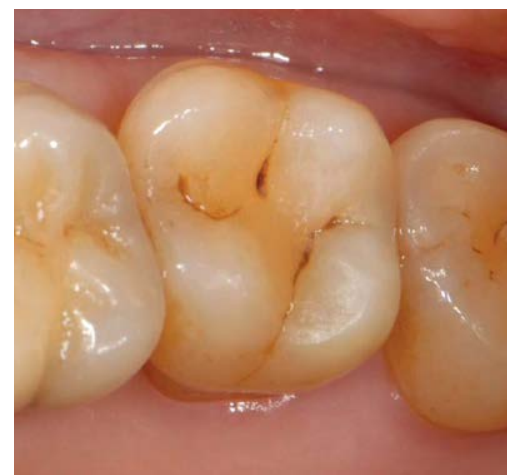

Abb. 2 Klinische Ausgangssituation: Sehr starke, lang anhaltende, spontan auftretende Schmerzen am auf den Kältetest (Kohlendioxid) hypersensibel reagierenden Zahn 27 im Sinne einer irreversiblen Pulpitis, parodontale Situation unauffällig (keine erhöhten Sondierungstiefen, kein Bluten nach Sondieren).

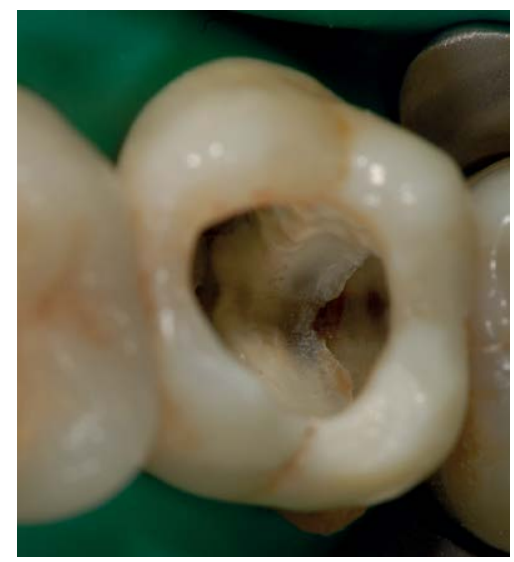

Abb. 4 Zahn 27 mit applizierter metallischer Teilmatrize. war eine Verkeilung nicht notwendig. Wäre die Teilmatrize nicht anlegbar gewesen, so hätte man zum Beispiel auch eine metallische Vollbandmatrize (Tofflemire) verwenden können. Durch Zurechtschneiden hätte man diese an den Defekt anpassen können. Eine Adaptation der Matrize am Zahn wäre durch das Zudrehen des Matrizenhalters möglich gewesen. Auch hier hätte man ohne Verkeilung gearbeitet. Wird auf eine Verkeilung verzichtet, so ist es insbesondere wichtig, dass man ein „Überstopfen“ des Kompositmaterials in den Zahnzwischenraum vermeidet. Dies gelingt durch Verwendung von Flowkomposit. Zur Konditionierung der Zahnoberflächen war Phosphorsäure verwendet worden (Ultra-Etch, Ultradent Products, UP Dental GmbH, Köln), als Klebesubstanz ein Kombinationspräparat aus Primer und Adhäsiv (Optibond FL, Kerr GmbH, Rastatt). Als fließfähiges Material kam ein Flowkomposit zum Einsatz (TetricEvo Flow, IvoclarVivadent, Ellwangen), das als erste dünne Schicht in die Kavität eingebracht worden war, um einerseits ein Überstopfen zu vermeiden, und anderer- 


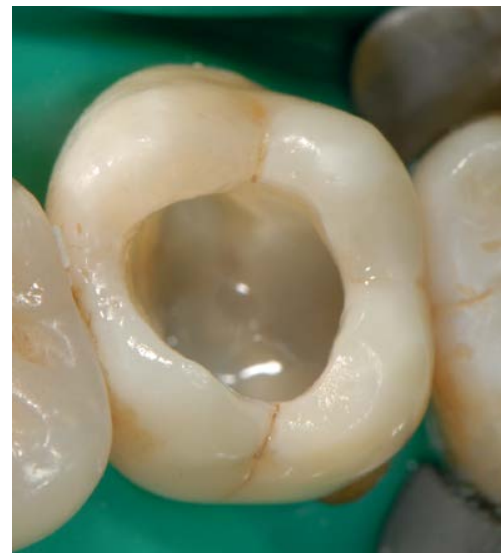

Abb. 5 Schichtweiser Verschluss der Kavität von okklusal nach interner Versorgung der distalen Wand und Vitelexstirpation. Die 1. fließfähige Schicht Komposit ist in den Zahn 27 eingebracht und lichtpolymerisiert.

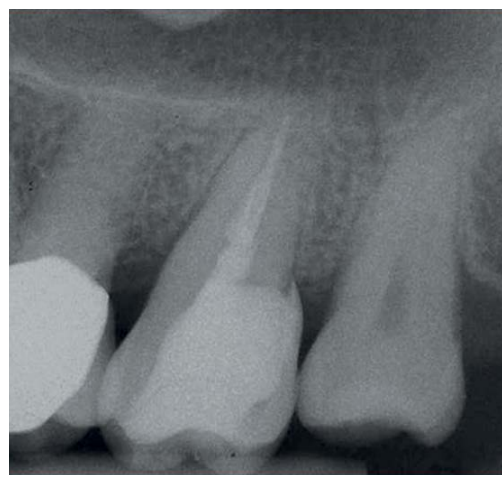

Abb. 7 Postoperatives Röntgenbild der Kompositrestauration an Zahn 27.

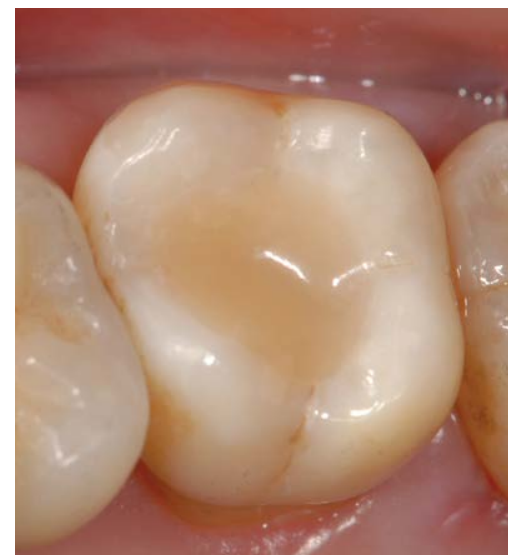

Abb. 6 Fertige Kompositrestauration an Zahn 27.

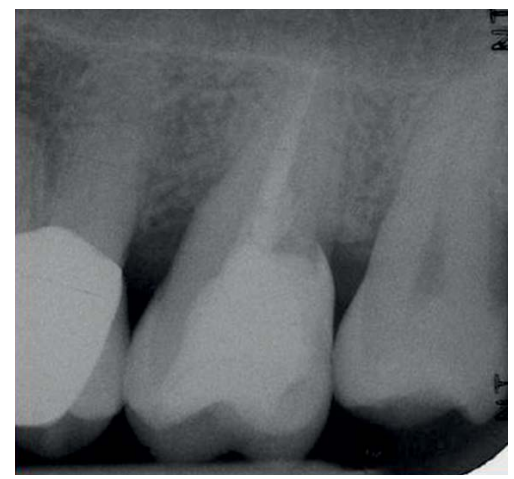

Abb. 8 Röntgenkontrollaufnahme nach 1,5 Jahren.

seits mögliche Unterschnitte und schwer zugängliche Bereiche ausfließen zu lassen ( $\bullet$ Abb. 5). Nach Aushärtung dieser 1. Schicht ist das Risiko des oben erwähnten Überstopfens mit viskösem Material in den Zahnzwischenraum verringert. Schließlich erfolgte die schichtweise Rekonstruktion der Kavität mittels viskösem Restaurationskomposit (TetricEvoCeram, IvoclarVivadent). Abschließend wurde die Okklusion adjustiert und eine Politur durchgeführt ( $\bullet$ Abb. 6 ). Die röntgenologische Kontrolle zeigte, dass ein gleichmäßiger und stufenfreier Übergang zwischen Kompositmaterial und Zahn erreicht wurde ( $\bullet$ Abb. 7). Die Patientin wurde anschließend hinsichtlich der Anwendung von Interdentalraumbürstchen instruiert und trainiert. Eine röntgenologische Kontrollaufnahme nach 1,5 Jahren zeigte einen intakten formschlüssigen Übergang zwischen Kompositmaterial und Zahnhals ( $\bullet$ Abb. 8).

Im vorliegenden Fall kam es zu einer Verletzung der biologischen Breite im Bereich der tief subgingival reichenden Kompositrestauration. Auf dem Verlaufsröntgenbild erkennt man, dass sich das knöcherne Niveau über 18 Monate circa $1 \mathrm{~mm}$ unterhalb des Restaurationsrandes eingestellt hat. Eine optimale häusliche Hygiene in

diesem Bereich war sicherlich von größter Bedeutung für die Etablierung stabiler parodontaler Verhältnisse. Die Kontrolluntersuchung der völlig beschwerdefreien Patientin zeigte keine erhöhten Sondierungstiefen und kein Bluten nach Sondieren. An dieser Stelle soll auf die alternative Behandlung verwiesen werden. Das Vorhandensein eines Kronenrandes bereitet im subgingivalen knochennahen Bereich zum Teil Schwierigkeiten. Es ist anspruchsvoll in diesem Bereich eine saubere Präparationsgrenze herzustellen und abzuformen, und es ist weiterhin anspruchsvoll in diesem Bereich einen stufen- und spaltfreien Kronenrand herzustellen. Des Weiteren wäre die Irritation der biologischen Breite durch einen Kronenrand wahrscheinlich großflächiger, da die Präparationsgrenze und damit der Kronenrand distal komplett abgesenkt würde und erst in den Übergängen nach oral und vestibulär wieder nach paragingival geführt werden könnte. Bei der Kompositrestauration beschränkt sich der subgingivale Anteil auf eine kleinflächige „Einkerbung“ ( $\bullet$ Abb. 4), die in die Tiefe reicht. Man kann mutmaßen, dass hier der kleinflächige Umfang des Kompositrandes, dessen stufen- und spaltfreier Übergang und eventuell die Oberflächenbeschaffenheit des Materials zu einer solch guten Toleranz geführt hat. Eventuell wäre der Rückgang des parodontalen Attachments nach Platzierung eines Kronenrandes deutlicher ausgefallen. Letztlich sind die Gründe für die unterschiedlichen Reaktionen auf Verletzungen der biologischen Breite jedoch bislang unbekannt [5].

\section{Fallbeispiel 2}

Direkte Kompositaufbauten nach

Entfernung von Kronen

Die Patientin stellte sich im Jahr 2003 mit dem Wunsch nach Verbesserung des ästhetischen Erscheinungsbildes ihrer oberen Frontzähne vor (○ Abb. 9). Das zahnärztliche Gespräch ergab, dass sie sich vor allem an den grauen Kronenrändern und der ungenügenden Hygienefähigkeit durch die vorliegenden positiven Stufen im Bereich der Kronenränder störte. Es lagen VMK-Kronen an den Zähnen 12-22 vor, die vor ca. 15 Jahren alio loco angefertigt worden waren. Aufgrund der unklaren endodontischen und parodontologischen Prognosen der Zähne wurde von einer Neuanfertigung der indirekten Kronen abgesehen. Alternativ wurde der Patientin vorgeschlagen, die alten Kronen abzunehmen und, bei genügender Restzahnsubstanz, die Zähne mittels direkten Kompositaufbauten zu rekonstruieren. Die Patientin war mit dem Behandlungsvorschlag einverstanden. Auf die endodontischen und parodontologischen Befunde, die zu der obigen Therapienentscheidung geführt haben, wird im Folgenden nicht eingegangen, da sie den Rahmen dieses thematisch fokussierten Fortbildungsartikels überschreiten würden. 


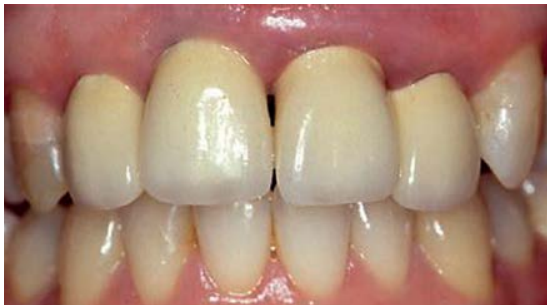

Abb. 9 Ausgangssituation mit VMK-Kronen an den Zähnen 12-22.

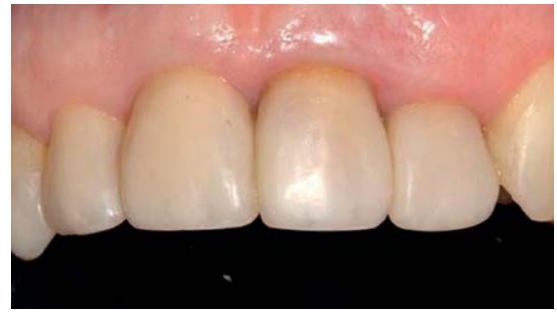

Abb. 12 Abschließende Situation nach Rekonstruktion der Zähne 12-22 mittels direkten Kompositaufbauten.

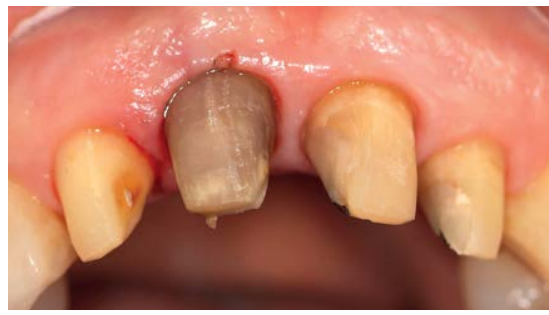

Abb. 10 Klinische Situation nach Entfernung der VMK-Kronen an 12-22.

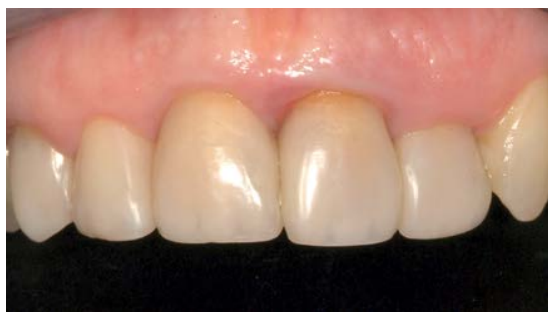

Abb. 13 Klinische Situation nach 4 Jahren.

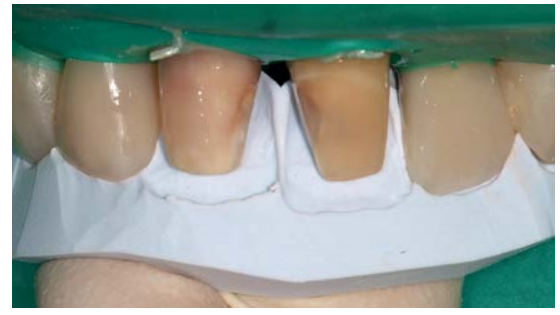

Abb. 11 Rekonstruktion der Zähne 11 und 21 mittels eines Silikonschlüssels.

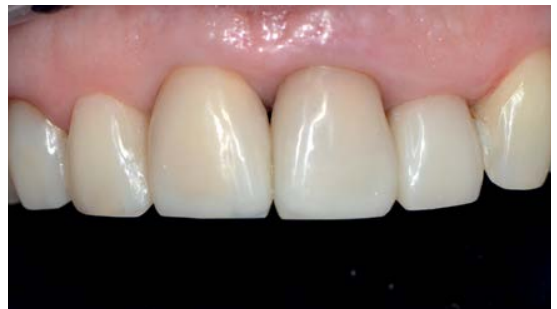

Abb. 14 Klinische Situation nach 10 Jahren und durchgeführter Farbkorrektur und Anpassung der Ebene der Inzisalkanten.
Nach Entfernung der alten Kronen zeigte sich der Zahn 11 stark verfärbt ( $\triangle$ Abb. 10). Deswegen wurde als Vorbehandlung eine interne Bleichung des Zahns (Natriumperborat $+\mathrm{H}_{2} \mathrm{O}$, einmalige Einlage) vorgenommen, die zu einer deutlichen Aufhellung des Zahnstumpfes führte. Aufgrund der Tatsache, dass der Zahn 11 einer Vorbehandlung unterzogen werden musste, wurde die Rekonstruktion der Frontzähne mit direkten Kompositaufbauten an den Zähnen 12 und 22 begonnen. Aus Gründen der Symmetrie ist es ansonsten anzuraten, die Rekonstruktion immer von der Mittellinie aus, also mit den Zähnen 11 und $21 \mathrm{zu}$ beginnen. Nach Aufbau der Zähne 12 und 22 in einer 1 . Sitzung erfolgte dann, nach erfolgreicher Bleichung, die Rekonstruktion von 11 und 21. Das Anlegen von Kofferdam ist eine hilfreiche Maßnahme, um das Arbeitsfeld und die Zahnoberflächen vor Kontaminierung zu schützen, vor allem, weil die Aufbauten bis leicht subgingival reichten. Zur Fixierung des Kofferdams an den konisch zulaufenden Zahnstümpfen wurden Ligaturen verwendet. Anschließend wurden die Zahnoberflächen angeraut (Mikrosandstrahlgerät, Kaltenbach \& Voigt, Biberach; $50 \mu \mathrm{m} \mathrm{Al}_{2} \mathrm{O}_{3}$ ), konditioniert (Ultra-Etch, Ultradent Products) und mit einem Adhäsivsystem (Optibond FL) behandelt. Es erfolgte jeweils der Aufbau der palatinalen Schmelzwand mithilfe eines Silikonschlüssels ( $\bullet$ Abb. 11). Anschließend wurden mesial und distal der Zähne Verschalungen [6, 7] mittels transparenter Kunststoffmatrizen und provisorischem Komposit durchgeführt. Nach Aufbau der mesialen und distalen Schmelzwände wurden die bukkalen Facetten rekonstruiert. Hier kam ein ästhetisch hochwertiges Kompositsystem (Enamel HFO, Micerium,
Avegno, I) mit Schmelz-, Dentin- und Charakterisierungsfarben zum Einsatz. Schließlich erfolgte die Ausarbeitung, wobei vor allem die Ränder der Aufbauten im Bereich der Gingiva exakt geglättet wurden. Dafür wurden sichelförmige Skalpelle (Nr. 12, 10-155-12 Nr. 12, Carl Martin $\mathrm{GmbH}$, Solingen), feine Diamantspitzen (8889 314 010, Komet Dental, Gebr. Brasseler GmbH \& Co. KG, Lemgo), und Finierstreifen (1965N, 3MESPE Deutschland $\mathrm{GmbH}$, Seefeld) verwendet. Schließlich erfolgte die Adaptation der Okklusion und Hochglanzpolitur. Die Behandlung wurde im Jahr 2004 abgeschlossen und die Patientin war zufrieden mit dem ästhetischen Erscheinungsbild und der Hygienefähigkeit der Restaurationen ( $\bullet$ Abb. 12). Sie wurde anschließend im Recall betreut, wobei sie Termine etwa einmal jährlich wahrnahm. Im Jahr 2008 stellte sich die Patientin mit einer verschlechterten parodontalen Situation an Zahn 21 vor, durch erhöhten Lockerungsgrad war es zu einer leichten Elongation und zum Rückgang der Gingiva an 21 gekommen ( Abb. 13). Die Patientin wurde im Folgenden in kurzen Recall-Abständen parodontologisch betreut und es erfolgte eine Politur der Restaurationen an 12-22. Sechs Jahre später, im Jahr 2014, zeigten sich stabile parodontologische und restaurative Verhältnisse. Die Patientin hatte in der Zwischenzeit mehrere häusliche Bleichversuche mit frei verkäuflichen Produkten durchgeführt und es war zu einer Aufhellung der Zähne im Unterkiefer gekommen. Deswegen äußerte sie den Wunsch einer Farbkorrektur der Restaurationen an den Zähnen 12, 11, 21 und 22, um diese farblich an die unteren Schneidezähne anzupassen. Des Weiteren wünschte die Patientin einen harmonischeren Schneidekantenverlauf mit weniger 
prominenten Eckzahnspitzen. Diesem Wunsch konnte entsprochen werden, indem in einer Sitzung Farb- und Formkorrekturen durchgeführt wurden. Dabei wurde das alte Kompositmaterial von den Fazialflächen der Zähne 12-22 schonend entfernt und durch einen helleren Farbton ersetzt. Dies erfolgte im Sinne der technischen Vorgehensweise einer Reparaturrestauration mittels Komposit. Anschließend wurde durch weitere additive (im Sinne von Reparaturrestaurationen mittels Komposit) und subtraktive Maßnahmen eine Harmonisierung des Schneidekantenverlaufes herbeigeführt ( $\odot$ Abb. 14). Abschließend lässt sich sagen, dass die Kompositaufbauten zur Rekonstruktion der Zahnstümpfe nach Entfernung von Kronenversorgungen in diesem Fall über 10 Jahre ein sehr gutes Überleben zeigten. Es wurden keine unerwünschten Ereignisse, wie zum Beispiel Frakturen oder Verfärbungen beobachtet. Aufgrund einer unsicheren parodontalen und endodontalen Ausgangssituation war ein Vorgehen gewählt worden, das es erlaubte, auf den weiteren Verlauf flexibel zu reagieren. In diesem Fall musste den parodontologisch begründeten Veränderungen der Zahnstellung und der RotWeiß-Ästhetik (Elongation und Gingivarückgang Zahn 21) sowie den durch Bleichmaßnahmen der Antagonistenzähne erfolgten Farbänderungen Rechnung getragen werden. Die Korrekturen konnten zeit- und kosteneffizient mittels additiver (Reparaturrestauration) und subtraktiver Maßnahmen umgesetzt werden. Dies wäre mit einer konventionellen prothetischen Versorgung mittels indirekt hergestellter Werkstücke kaum realisierbar gewesen.

\section{Diskussion}

Die vorgestellten Fälle zeigten mögliche Behandlungsalternativen bei der Versorgung ausgedehnter und tief subgingivaler Zahnhartsubstanzdefekte. Derartige Behandlungen gehören bislang nicht zur Standardversorgung im zahnmedizinischen Alltag, stellen aber nach Meinung der Autoren sinnvolle Ergänzungen im Therapiespektrum ausgedehnter Defekte dar.

Eine kritische Evaluation der Behandlungsmethoden zeigt bislang noch folgende nachteilige Aspekte auf:

- Es liegen nur begrenzte Studiendaten zum Langzeitüberleben vor.

Korrespondenzadresse PD Dr. Diana Wolff Poliklinik für Zahnerhaltungskunde

Klinik für Mund-, Zahn- und Kieferkrankheiten

Universitätsklinikum Heidelberg Im Neuenheimer Feld 400 69120 Heidelberg diana.wolff@med.uni-heidelberg.de

- Das Ergebnis und der Erfolg der Behandlung werden maßgeblich von der technischen Vorgehensweise beeinflusst.

- Bei Verletzung der biologischen Breite ist bislang unklar, welche Auswirkungen sich im individuellen Fall auf das Parodont und den Knochen ergeben.

- Der Aufwand, der für die Herstellung derartiger Restaurationen geleistet werden muss, ist bisher nicht im Leistungskatalog der Krankenkassen adäquat abgebildet.

Nicht von der Hand zu weisen sind jedoch auch die Vorteile, die sich bei Verwendung der beschriebenen Techniken ergeben:

- Bestehende Restzahnsubstanz kann meistens vollständig erhalten werden.

- Eine Reparatur der Restaurationen ist einfach und zeiteffizient möglich.

- Jede andere Versorgung (z.B. indirekte Kronenversorgung oder Veneers) kann zu einem späterem Zeitpunkt noch durchgeführt werden.

- Es ist möglich, auf unplanbare oder unerwartete Ereignisse flexibel zu reagieren.

- Die Behandlung ist durch Wegfall labortechnischer Leistungen vergleichsweise kosteneffizient.

\section{Zusammenfassung}

Die vorgestellten Vorgehensweisen sind techniksensitiv und anspruchsvoll in der Umsetzung. Bei korrekter Durchführung lassen sich jedoch funktionell, biologisch und ästhetisch gute Ergebnisse erzielen. Die klinische Erfahrung zeigt, dass bei guter Pflege und Reinigung durch den Patienten mit einem guten Überleben zu rechnen ist. Das Spektrum zur Versorgung ausgedehnter und tief subgingivaler Defekte lässt sich mit Anwendung der vorgestellten Techniken erweitern, sodass auch Problemfälle, oder Zähne mit unklarer Prognose gut und langfristig versorgt werden können.

Interessenkonflikt: Es besteht kein Interessenkonflikt.

Literatur

1 Fennis WM, Kuijs RH, Roeters FJ et al. Randomized control trial of composite cuspal restorations: fiveyear results. J Dent Res. 2014; 93: 36-41

2 Frese C, Schiller $P$ et al. Recontouring teeth and closing diastemas with direct composite buildups: a 5-year follow-up. J Dent. 2013; 41: 979-985

3 Naumann M, Preuss A, Frankenberger R. Reinforcement effect of adhesively luted fiber reinforced composite versus titanium posts. Dent Mater 2007; 23: 138-44

4 Micheelis W, Schiffner U. Vierte Deutsche Mundgesundheitsstudie (DMS IV). Köln: Institut der Deutschen Zahnärzte, 2006

5 Frese C, Wolff D, Staehle HJ. Proximal box elevation with resin composite and the dogma of biological width: clinical R2-technique and critical review. Oper Dent. 2014; 39: 22-31

6 Klaiber B. Alles noninvasiv - Zahnformveränderung, Lückenschluss, Reduktion schwarzer Dreiecke. zm 2006; 96: 52-59

7 Hugo B, Denner W. Approximaler Zahnaufbau zum Diastemaschluss. BioStyle Arbeitsbuch : Das Ästhetik-Komposit System: Dreve Dentamid; 2005. p. 32-42 


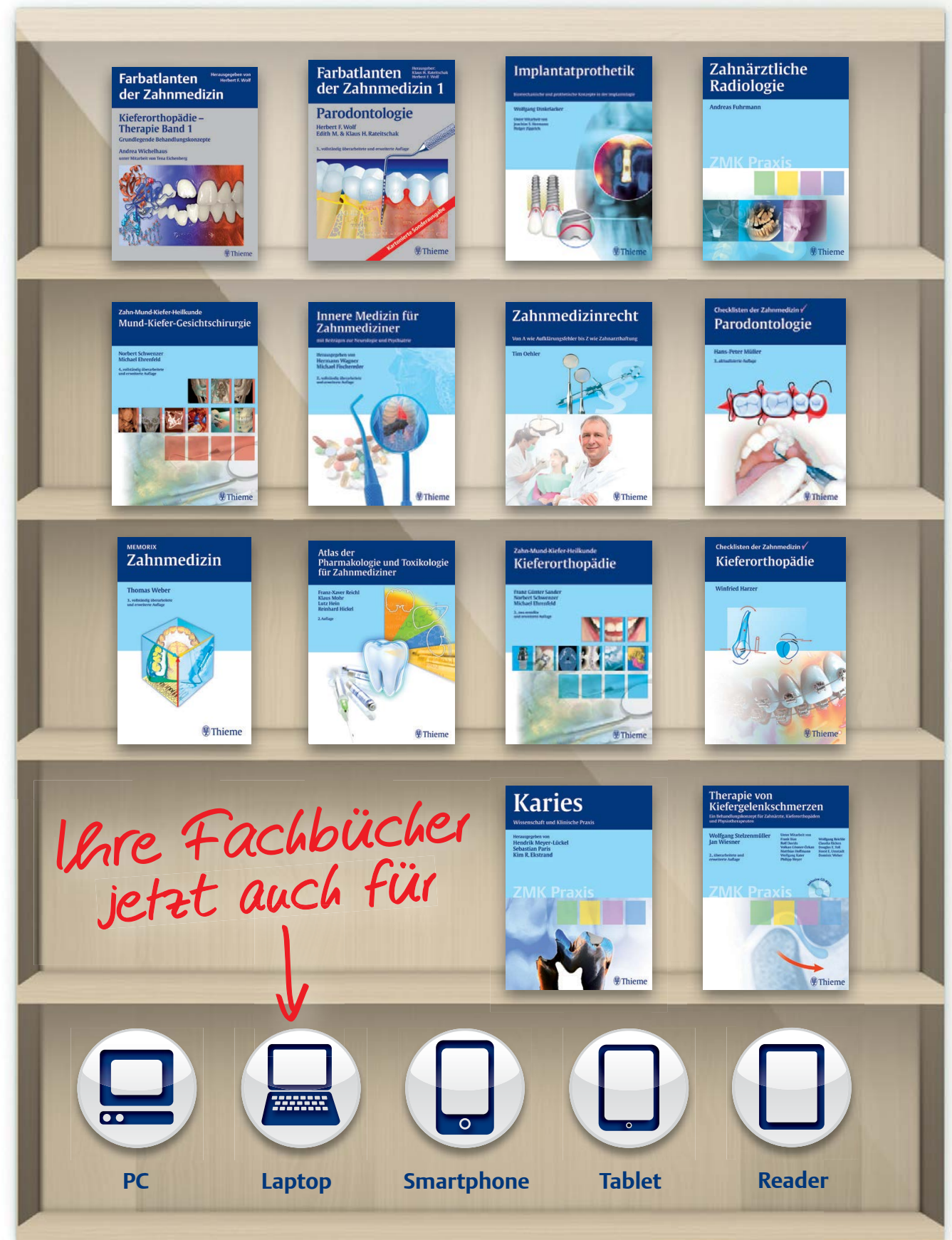

Viele Bücher gibt es jetzt als E-Book, sowohl als PDF wie auch als ePub.

Informieren Sie sich einfach unter 